\title{
COMETH: a CMOS pixel sensor for a highly miniaturized high-flux radiation monitor
}

\author{
Yang Zhou ${ }^{1}$, Jérôme Baudot, Christine Hu-Guo, Yann Yu, Kimmo Jaaskelainen and \\ Marc Winter \\ IPHC/CNRS, Université de Strasbourg \\ 23 rue du loess, BP 28, F-67037 Strasbourg, France \\ E-mail: yang.zhou@iphc.cnrs.fr, jerome.baudot@iphc.cnrs.fr, \\ christine.hu@iphc.cnrs.fr, Yann.Hu@iphc.cnrs.fr, \\ Kimmo.Jaaskelainen@iphc.cnrs.fr, Marc.Winter@iphc.cnrs.fr
}

The need for miniaturized and versatile real-time radiation monitors has become a general trend for spacecraft applications. It requires a highly integrated detection system with the ability to count and identify ion species in a high-flux with mixed particles environment. We have proposed a new strategy to meet these requirements with a single CMOS pixel chip.

This sensor is based on a matrix of $50 \times 50 \mu \mathrm{m}^{2}$ pixels, read out in rolling-shutter mode, and features columns ended by 3-bit ADCs with tuneable threshold. An embedded digital algorithm extracts the particle properties from the pixel information to provide the radiation flux online.

A reduced scale prototype with $32 \times 32$ pixels and 32 column-level ADCs has been designed and fabricated in a $0.35 \mu \mathrm{m}$ CMOS process. The layout of the identifying and counting algorithm, downstream the pixel matrix, was developed in the same process. A full simulation of this layout for a subset of columns was used to check the algorithm output against a variety of inputs.

The prototype was tested with X-rays, $\beta$ - particles and laser illumination. Results confirm previous simulations addressing gain and linearity. Column-level ADCs also perform as expected. Those measurements validate the possibility to monitor proton and electron fluxes up to $10^{7}$ particles $\mathrm{cm}^{-2} \cdot \mathrm{s}^{-1}$ and to distinguish protons from electrons for energies lower than $50 \mathrm{MeV}$. A brief review of this first prototype design and tests is presented in this paper.

Technology and Instrumentation in Particle Physics 2014

2-6 June, 2014

Amsterdam, the Netherlands

${ }^{1}$ Speaker. 


\section{Introduction}

The Earth orbit environment is populated by a great variety of energetic charged particles which are dominated by protons and electrons [2, 3]. Their total omnidirectional fluxes may reach several $10^{7} \cdot \mathrm{cm}^{-2} \cdot \mathrm{s}^{-1}$ with energies covering many orders of magnitude (for protons in the range 0.1 - $400 \mathrm{MeV}$, and for electrons in the range $0.04-7 \mathrm{MeV}$ ).

CMOS Pixel Sensors (CPS), being monolithic full detection systems, have the potential to provide real-time measurement of this high flux environment characterized by mixed particle types with additional advantages, including low power and weight, as well as small size. We have proposed [1] a CPS architecture named $\mathrm{COMETH}^{2}$, which includes a $64 \times 64$ square pixel matrix with $50 \mu \mathrm{m}$ pitch size, 64 column-level 3-bit ADCs and an embedded digital processing logic to directly provide the impinging particle flux information by their species and energies. The pixelization of the sensitive part helps in increasing both the counting rate and the measurable energy range. Simulations have validated this concept and indicated COMETH could count up to $10^{7}$ particles $\cdot \mathrm{cm}^{-2} \cdot \mathrm{s}^{-1}$ with marginal error due to particles pileup or clusters merging, and could separate electrons from protons up to the energy of about $50 \mathrm{MeV}$ [1].

\section{Review of the COMETH design}

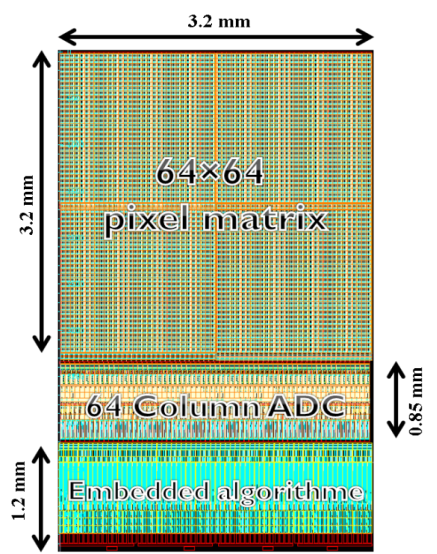

(a)

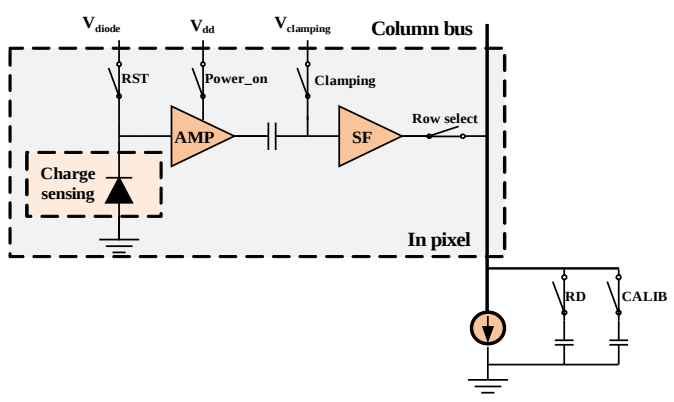

(b)

Figure 1: Layout of COMETH (a) and the architecture of the pixel (b).

Figure 1 (a) displays the layout of COMETH; it features a sensitive area of about $10 \mathrm{~mm}^{2}$, while the total area comprising the micro-circuits for data processing reaches about $17 \mathrm{~mm}^{2}$. A single $4.3 \times 4.3 \mu^{2}$ n-well/p-epi sensing diode located in the center of each pixel collects the charges generated in the sensitive layer of the sensor. Still within the pixel, the sensing diode signal goes through a pre-amplification stage and undergoes Correlated Double Sampling (CDS) (Fig. 1 (b)) based on clamping. The useful signal is calculated as the difference (for each pixel) between two samplings. Next, outside the sensitive area, column-level ADCs digitize with 3 bits accuracy the signals of an entire row. The final digital processing occurs in an embedded algorithm integrated on the chip. COMETH is designed to operate in a rolling-shutter

\footnotetext{
${ }^{2}$ COunter for Monitoring the Energy and Type of charged particles in High flux.
} 
mode reaching a 65,000 frames/s read-out speed. Its total estimated power dissipation is about $100 \mathrm{~mW}$ (under $3.3 \mathrm{~V}$ power supply).

The above features result from an optimization conducted with a previous simulation study, reported in [1], to guarantee robust flux estimation by species up to $10^{7}$ particles $\mathrm{cm}^{-2} \cdot \mathrm{s}^{-1}$. In particular, the sensor response model used, predicted individual pixel charges from a hundred $\mathrm{e}^{-}$to 4400 e; electron cluster size between 2 to 3 pixels; and low energy protons firing sub-area as large as $9 \times 9$ pixels with the cluster size being inversely proportional to the incident energy. The design of the three main signal processing stages was driven by these expectations.

The in-pixel pre-amplification stage features a gain and noise to match both the detection sensitivity around $100 \mathrm{e}^{-}$signal and the linearity range up to $4000 \mathrm{e}^{-}$charges.

The column ADC was designed using the SAR (successive approximation register) architecture. It has a tunable threshold. However, with respect to usual SAR ADC, the one in COMETH performs an extra comparison before the analog to digital conversion; the discrimination threshold is tuneable externally in order to adjust the detection sensitivity. When this first comparison fails, the ADC turns itself off until the next sample. This strategy allows taking advantage of the low occupancy of the matrix (more than $97 \%$ of the pixels are unfired up to a flux as high as $10^{7}$ particles $\cdot \mathrm{cm}^{-2} \cdot \mathrm{s}^{-1}$ ) in order to control the system power dissipation.

The last stage consists in the embedded algorithm, which processes the ADCs digital outputs. Its functionalities include: clustering of adjacent pixels, summation of their digitized signal and decision to increment the specific counter corresponding to a given energy range and species. Indeed, a memory containing several counters, one per energy and species, forms the final result of the sensor. This memory can then be read out at a low frequency (Hertz range) to extract the information from the sensor with a low data throughput as usually required for small satellites [4].

This digital processing stage has been described using Verilog (hardware description language) and synthesized in a $0.35 \mu \mathrm{m}$ process. The corresponding microcircuits occupy a $1.2 \times 3.2 \mathrm{~mm}^{2}$ layout size, and dissipate $56.7 \mathrm{~mW}$ power (with $3.3 \mathrm{~V}$ power supply) according to the simulation estimate. A full simulation of the layout against many inputs was realized to estimate its performances. The simulation validates its ability to reconstruct most of cluster cases. Failure only occurs in the presence of several adjacent dead pixels, which leads to the reconstruction of single impact as two events. This case is however expected to be marginal.

\section{Test results of the first prototype}

A reduced scale prototype with $32 \times 32$ pixels and 32 column ADCs was fabricated in a $0.35 \mu \mathrm{m}$ process, and tested to validate the COMETH concept. Dedicated analogue outputs for each column, bypassing the ADC, were foreseen in order to characterize in detail the pixel response.

The pixel matrix was illuminated with three types of radiations: monochromatic X-rays to obtain the charge-to-voltage conversion factor (CVF) and the charge collection efficiency (CCE); Beta electrons to assess the sensitivity to minimum ionizing particles with the signalover-noise ratio (SNR) and the cluster shape; and finally infrared photons from a focalized laser 
beam to test the pixel response linearity over the relevant signal magnitude range, the sensing diode reset, and recovery efficiency.

The end column ADCs were tested with external bias voltage replacing the pixel outputs. Their noise performances, transfer function, power dissipation and tunable threshold functionality have also been measured.

\subsection{Test with a ${ }^{55} \mathrm{Fe}$ source}

A ${ }^{55} \mathrm{Fe}$ radioactive source was used to illuminate the prototype with mostly $5.9 \mathrm{keV} \mathrm{X}$ rays, which generate about $1640 \mathrm{e}$. Within each read-out frame, a few impacts are reconstructed individually as groups of adjacent fired pixels. For each of these clusters, the seed pixel is defined as the one with the highest signal.

Fig. 2 (a) shows the distribution of the charge collected by the seed pixel and digitized by an external 12-bit ADC. The most probable value (MPV) of the distribution was estimated with a gaussian fit in the area of the distribution maximum; it amounts to 114.9 \pm 0.4 ADC units. A calibration peak is expected at the far end of the spectrum. It corresponds to events where the Xrays convert nearby or within the sensing diode. In this situation, all the $1640 \mathrm{e}^{-}$generated by the photoconversion are collected by a single pixel, contrary to most clusters for which only a fraction of these charges is collected on the seed pixel. Therefore, to reproduce the spectrum tail, we used a two components fit, a linear distribution describing the multi-pixel clusters combined with a gaussian distribution describing the single pixel cluster (calibration events), see figure 2 (b). From the fit, the calibration peak is located at $364 \pm 3$ ADC units. The ratio of the distribution MPV to the calibration peak position yields that the CCE of the seed pixel amounts to (31.6 \pm 0.5$) \%$. Moreover, the CVF is found to amount to $33 \mu \mathrm{V} / \mathrm{e}^{-}$; which is obtained from the ratio of the corresponding analogue voltage value of the calibration peak at 364 ADC units with $1640 \mathrm{e}^{-}$.

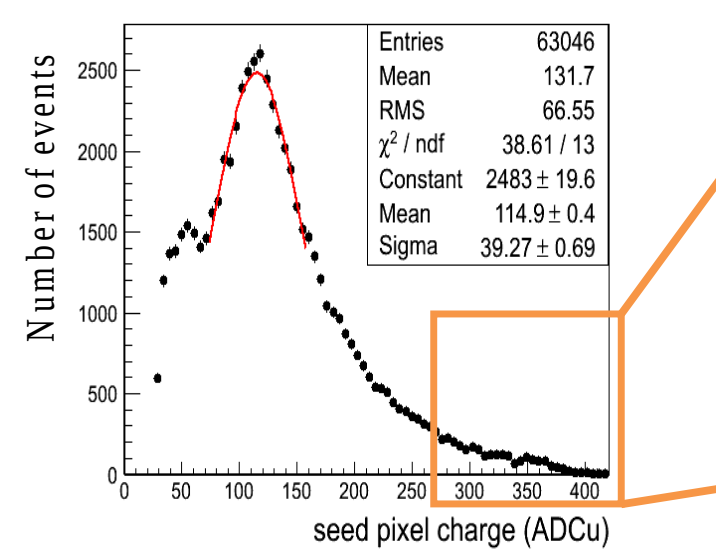

(a)

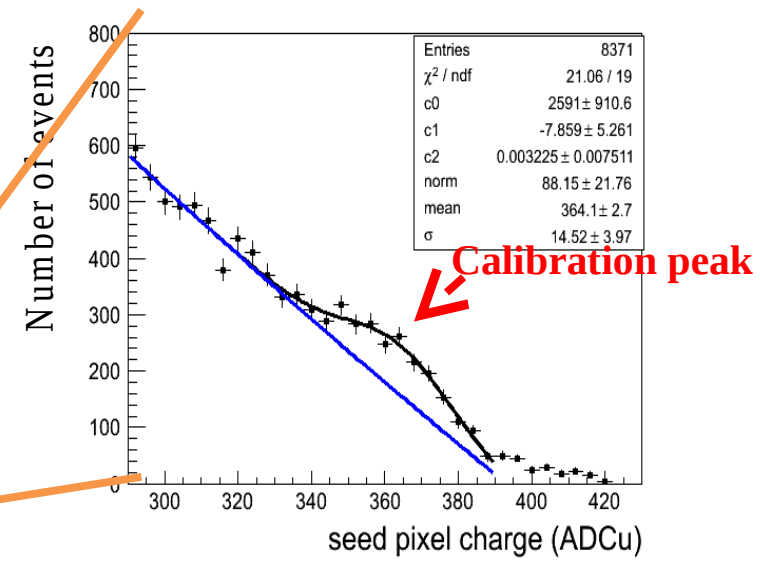

(b)

Figure 2: Collected charge distribution observed with a ${ }^{55} \mathrm{Fe}$ source. Fig. (a) shows the entire distribution while Fig. (b) displays a zoom on the calibration peak. See text for the fit explanations.

We also considered the sum of charges collected by the individual pixels within a cluster. Figure 3 displays the cluster charge collected by areas of $3 \times 3$ and $5 \times 5$ pixels centered on the 
seed pixel. The MPVs are again estimated from a gaussian fit restricted to the distribution maximum; they amount to $323 \pm 0.4$ ADC units and $343 \pm 1.0$ ADC units respectively. We conclude that the CCE for a $3 \times 3$ cluster is already as high as $(88.7 \pm 0.5) \%$ and that considering a larger cluster only marginally increases the total charge collected.

The test with ${ }^{55} \mathrm{Fe}$ demonstrated a satisfactory CCE for a pixel sensor which features a relatively large pitch size of $50 \mu \mathrm{m}$ combined with a single, relatively small, central sensing diode $\left(4.3 \times 4.3 \mu \mathrm{m}^{2}\right)$.

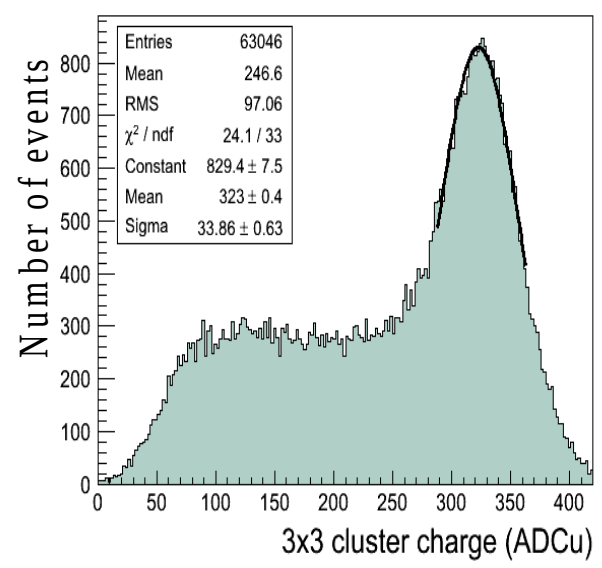

(a)

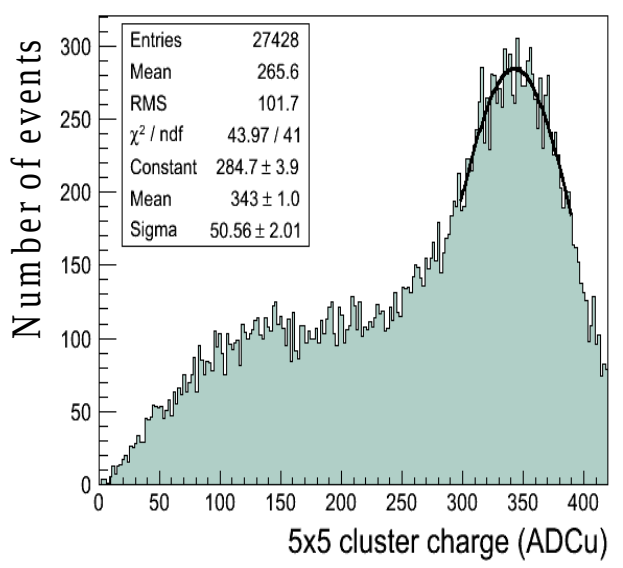

(b)

Figure 3: Cluster collected charge with the ${ }^{55} \mathrm{Fe}$ source for $3 \times 3$ pixels (a) and $5 \times 5$ pixels (b). The lines represent gaussian fits to estimate the MPV of the distribution.

\subsection{Test with a Beta minus source}

A ${ }^{90} \mathrm{Sr}$ source was used to provide electrons with an energy spectrum typical of $\beta$ emission, the end point being at $2.3 \mathrm{MeV}$. This range matches well the electron energy for the COMETH application, and represents the lowest ionization signal to be detected. The same treatment as in the case of $\mathrm{X}$-rays was applied to the analogue data to obtain the cluster information for each impinging $\beta$-.

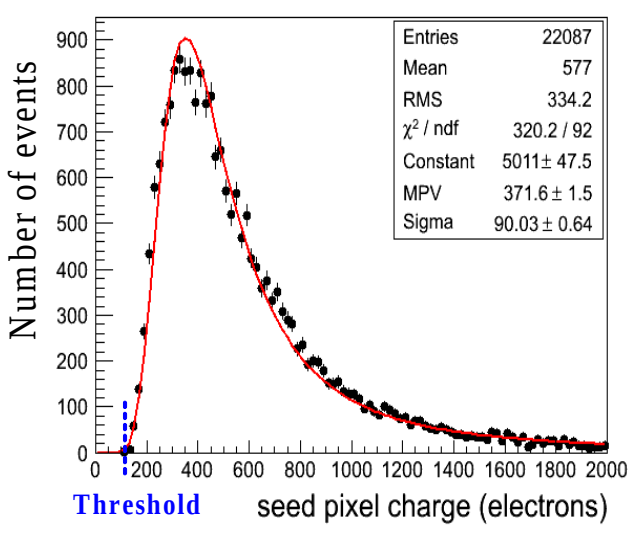

(a)

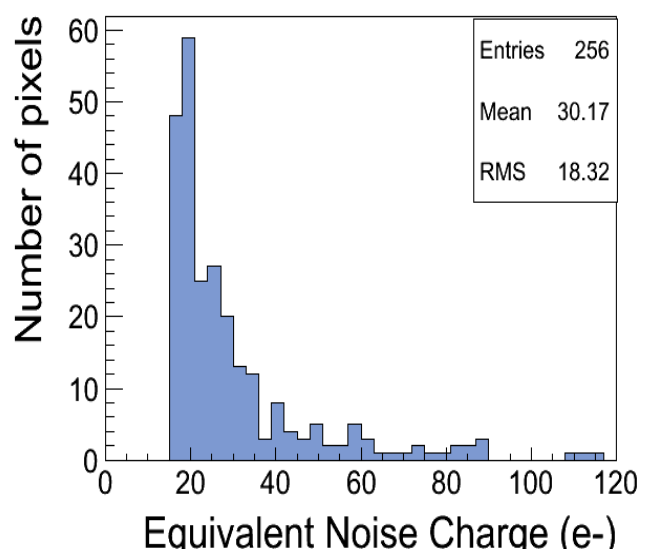

(b)

Figure 4: Seed pixel charge collected with a ${ }^{90} \mathrm{Sr} \beta$ - source (a) and individual pixel noise distribution (b). 
The seed pixel charge distribution is shown in Fig 4 (a); it is fit with a Landau function to account for the energy deposite fluctuations. The MPV is evaluated at $371.6 \pm 1.5 \mathrm{e}$. This value is well in accordance with the expectation from a minimum ionizing particle which generates about $80 \mathrm{e} / \mu \mathrm{m}$ [5] in average, taking into account the $14 \mu \mathrm{m}$ thickness of the sensitive volume for the $0.35 \mu \mathrm{m}$ CMOS process, and the charge collection efficiency (31.6 $\pm 0.5 \%)$ for the seed pixel determined with X-rays. In COMETH the digital conversion of the pixel signal is triggered above a given threshold, which hence drives the detection efficiency. From the integral of the seed pixel charge distribution, we note that for $99.95 \%$ of the impacts the seed pixel collects more than $120 \mathrm{e}$.

Moreover, the SNR is also an important parameter driving the detection efficiency. The individual pixel Equivalent Noise Charge (ENC) was estimated from the CDS value variation without any source. The average pixel noise corresponds to $30 \mathrm{e}^{-}$as indicated by the distribution of the individual pixel noises from Fig. 4 (b). The corresponding most probable SNR value is about 13.

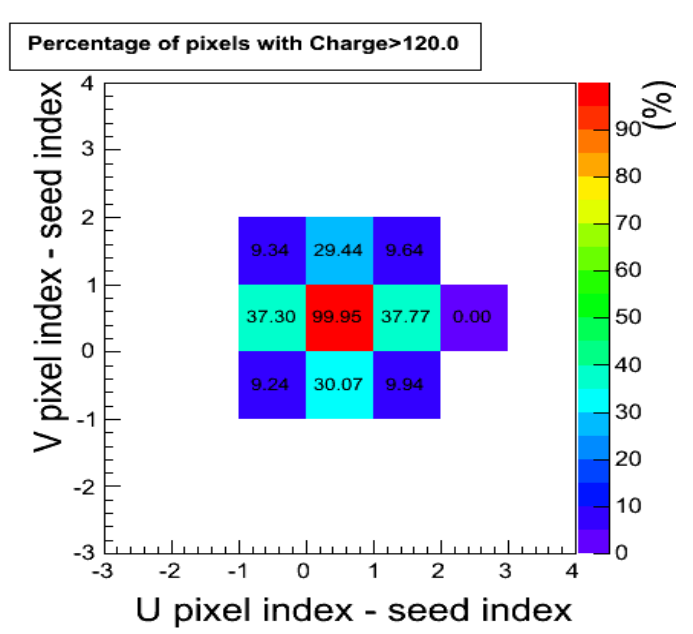

(a)

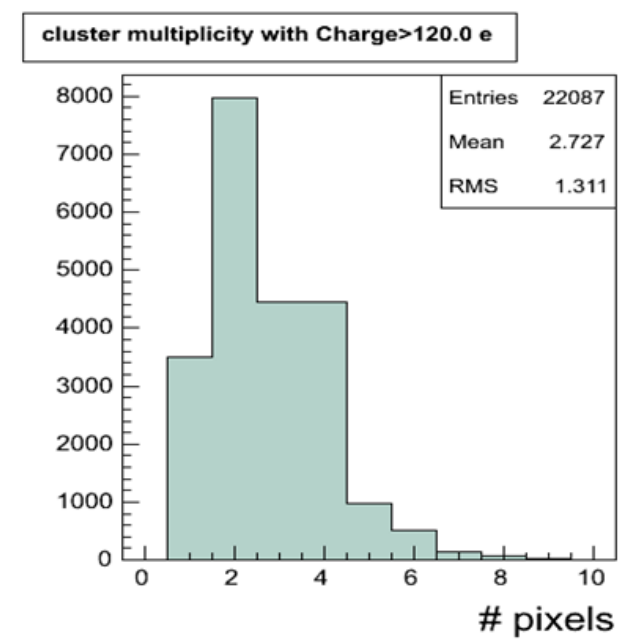

(b)

Figure 5: Percentage of pixels with charge above $120 \mathrm{e}^{-}$with a ${ }^{90} \mathrm{Sr} \beta$ - source (a) and cluster multiplicity of pixels with a charge $>120 \mathrm{e}^{-}$(b).

Fig. 5 illustrates the cluster shape obtained with electrons. For each cluster, we kept only the pixels with an analogue signal in excess of $120 \mathrm{e}$, since this threshold guarantees a detection efficiency above $99.9 \%$ as estimated before. Fig. 5 (a) depicts how often a given pixel is fired above this threshold in the $7 \times 7$ pixels subarea around the seed pixel. The total number of fired pixels per cluster is shown in Fig. 5 (b). We observe that the vast majority of clusters does not exceed 4 pixels in size, the average size being 2.7 pixels, as assumed in the pixel response model of our previous simulations [1].

\subsection{Test with an infrared laser}

The light of a laser diode with a $1063 \mathrm{~nm}$ wavelength was focused within a $5 \mu \mathrm{m}$ diameter spot on the sensor. In order to mimic a particle crossing the sensor, light was delivered through pulses much shorter than the integration time. 
The pluse length being fixed, the light intensity depends on two parameters: the input current to the laser diode and the number of pulses shot during one sensor integration time. To control the sensor response linearity, we varied this number of pulses for these different input currents (low, middle, high); so that the number of charges generated by the illumination is proportional to the number of pulses. Results are shown in Fig. 6. They indicate that the linearity response range of a single pixel extends up to $5000 \mathrm{e}$, complying with the requested value of $4400 \mathrm{e}^{-}$. The limitation observed originates from the saturation of the in-pixel amplifier.

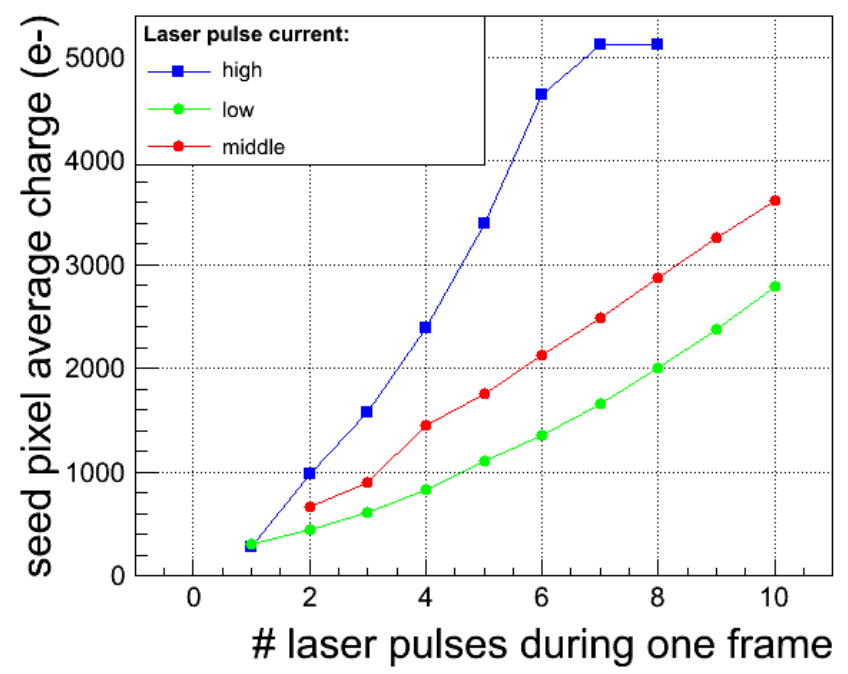

Figure 6: Evolution of the pixel response to a linear increase of the laser illumination, for three different laser currents. The laser illumination amplitude depends linearly on the number of laser pluses shot during one sensor frame.

\subsection{Test results of the ADC}

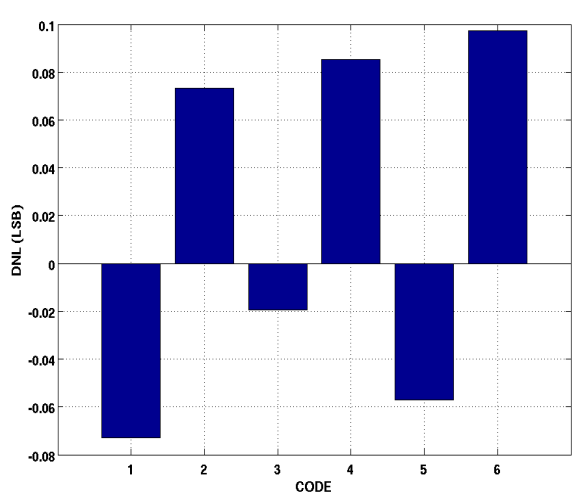

(a)

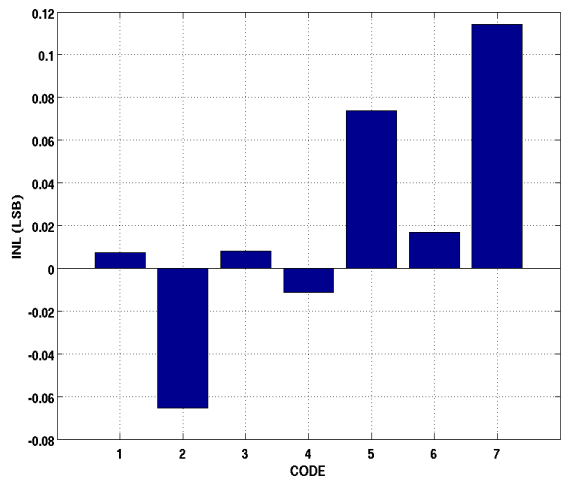

(b)

Figure 7: DNL (a) and INL (b) characteristics of one column ADC.

Column ADCs were tested with external input voltage replacing the pixel outputs. Their expected Least Significant Bit (LSB) is 700 e-, corresponding to $23.1 \mathrm{mV}$ taking into account the pixel CVF measured previously. This test results indicate that each single column has a good noise performance. The temporal noise (rms) is indeed only $0.02 \mathrm{LSB}$; the integral nonlinearity 
(INL) and the differential nonlinearity (DNL) are less than \pm 0.12 LSB illustrated by Fig. 7. However, the fix pattern noise (FPN) between columns of this prototype is currently too high: the rms value being $0.21 \mathrm{LSB}$, it prevents the proper operation of the pixel matrix coupled with the ADCs. The FPN is the main characteristic to improve in the next prototype, is foreseen to be mitigated by an order of magnitude.

The ADC power dissipation was also measured. It amounts to 532 and $759 \mu \mathrm{W}$ with an input signal respectively lower and higher than threshold. These results confirm that the design is power efficient due to the introduction of the threshold comparison. Because most of the pixels are below readout threshold during the operation, the average ADC power dissipation would be very close to the low power dissipation level without signal, for instance $539 \mu \mathrm{W} / \mathrm{ADC}$ with $3 \%$ fired pixels.

\section{Conclusions and perspectives}

A quarter-size prototype of the proposed CMOS pixel sensor for high-flux radiation counter has been designed and fabricated in a $0.35 \mu \mathrm{m}$ process. Tests of the pixel matrix corroborate the expectation from previous simulations [1] on the key parameters: charge collection efficiency (above 30\% of the seed pixel for X-rays), above 99.9\% detection efficiency for minimum ionizing particles (MIP), cluster size of a few pixels (average size being 2.7 pixels for MIP), and linearity of the response over the expected signal range (0 to $5000 \mathrm{e}^{-}$for a single pixel). Separate evaluation of the analogue to digital conversion stage over 3 bits yields integral and differential nonlinearities below 0.12 LSB. The embedded digital processing algorithm, which achieves the final flux evaluation online, was also synthesized and validated.

All these elements make us confident that a CMOS radiation counter with very low mass and power can indeed measure flux as high as $10^{7}$ particles $\cdot \mathrm{cm}^{-2} \cdot \mathrm{s}^{-1}$, as our previous simulation work indicated.

The steps needed before the possible production of the complete monitor include: measurement of the prototype sensor response to low energy protons; reduction of the pixel noise and of the ADC FPN between columns, and further optimization of the system power dissipation.

\section{References}

[1] Y. Zhou et al, CMOS Pixel Sensor for a Space Radiation Monitor with very low cost, power and mass, JINST 7 (2012) C12003.

[2] D. M. Sawyer and J. I. Vette, AP-8 trapped proton environment for solar maximum and solar minimum, 1976 NASA TM-X-72605.

[3] J. I. Vette, The AE-8 trapped electron model environment, 1991 NSSDC/WDC-A-R\&S 91-24.

[4] H. Helvajian and S.W. Janson, Small Satellites: Past, Present, and Future, The Aerospace Press and AIAA, Reston, Va, 2008.

[5] R. Turchetta et al, A monolithic active pixel sensor for charged particle tracking and imaging using standard VLSI CMOS technology, Nuclear Instruments and Methods in Physics Physics Research, Section A, Volume 458, Issue 3, p. 677-689, 2001. 